Pacific Journal of Mathematics

A PROBLEM OF DOUGLAS AND RUIN ON FACTORIZATION 


\section{A PROBLEM OF DOUGLAS AND RUDIN ON FACTORIZATION}

\section{J. BOURGAIN}

If $f$ is a bounded measurable function on the circle $\pi$, then $\int_{\pi} \log |f| d m>-\infty$ expresses the necessary and sufficient condition on $f \neq 0$ to be of the form $f=g \cdot \bar{h}$ where $g, h \in H^{\infty}$. This question was proposed by Douglas and Rudin in [1], where they approximate unimodular functions on $\pi$ by quotients of Blaschke products.

Introduction. $\pi$ stands for the circle group and $m$ its normalized invariant measure. $H^{\infty}$ will be considered as a (closed) subalgebra of $L^{\infty}=L^{\infty}(\pi)$. In [2], Douglas and Rudin consider the set $\tilde{Q}$ of these functions in $L^{\infty}$ which are of the form $\phi \bar{\psi}$ with $\phi, \psi H^{\infty}$-functions. They noticed that then, by Jensen's inequality if $f=\phi \bar{\psi} \neq 0$

$$
\int_{\pi} \log |f| d m=\int_{\pi}(\log |\phi|+\log |\psi|) d m>-\infty
$$

has to be true and asked whether this property was also sufficient. If $\log |f|$ in $L^{1}(\pi)$, we may define the outer function

$$
h(z)=\exp \left\{\int \log \left|f\left(e^{i \theta}\right)\right| \frac{e^{i \theta}+z}{e^{i \theta}-z} m(d \theta)\right\}
$$

for which $|h(z)|=|f(\theta)|$ if $z=e^{i \theta}$ a.e. Thus $h^{-1}\left(e^{i \theta}\right) f(\theta)$ is an unimodular function on $\pi$ and can be written as $e^{\imath a}$ where $a$ in $L^{\infty}(\pi)$ takes values in $[-\pi, \pi[$.

Proposition. There are Blaschke products $B_{1}, B_{2}$ such that

$$
\left\|\mathscr{H}\left[a-\operatorname{Arg} \frac{B_{1}}{B_{2}}\right]\right\|_{\infty}<c
$$

where $\mathscr{H}$ denotes the Hilbert-transform and $c$ is numerical. We consider Arg as $[-\pi, \pi[$ valued.

If $b=a-\operatorname{Arg} B_{1} / B_{2}$, we obtain the decomposition

$$
e^{i a}=B_{1} e^{i F} \widehat{B_{2} e^{-i F}}
$$

taking $F$ in $H^{\infty}$ with $b=2 \operatorname{Re} F$. This will imply the result stated in the abstract. 
Notice that as corollary $L^{\infty}=H^{\infty} \overline{H^{\infty}}+\mathrm{C}$ and $f$ in $L^{1}(\pi)$ belongs to $H^{2} \overline{H^{2}}$ iff $\log |f|$ in $L^{1}$ (or $f=0$ ).

To verify the first assertion, let $f \in L^{\infty}$ and define $g_{\theta}=f+e^{\imath \theta}$. Then, applying Jensen's inequality in the $\theta$-variable

$$
\int\left\{\int \log \left|g_{\theta}\right|\right\} m(d \theta)=\iint \log \left|f(\psi) e^{-i \theta}+1\right| m(d \theta) m(d \psi) \geq 0 .
$$

Hence $\int \log \left|g_{\theta}\right| m(d \theta) \geq 0, g_{\theta} \in H^{\infty} \overline{H^{\infty}}$ for some $\theta$.

The second property is seen by writing

$$
f=f e^{-a} F \bar{F}
$$

where $a=\log |f|$ is in $L^{1}, F=\exp \frac{1}{2}(a+i \mathscr{H}[a])$ is the boundary value of an $H^{2}$-function. Finally $f e^{-a}$ is again unimodular and hence in $H^{\infty} \overline{H^{\infty}}$.

Proof of Proposition. The argument is constructive. It will be based on $L^{1}$-approximation of $L_{R}^{\infty}$ functions by elements of $\operatorname{Re} H^{\infty}$ and the constructive proof given by $\mathrm{P}$. Jones of the Douglas-Rudin approximation theorem (see [3]).

LEMMA 1. If $a$ in $L_{R}^{\infty}(\pi)$ and $\varepsilon>0$, there is $b$ in $L_{R}^{\infty}(\pi)$ satisfying $\|a-b\|_{1}<\varepsilon\|a\|_{\infty}$ and $\|b\|_{\infty}+\|\mathscr{H}[b]\|_{\infty}<c_{1} \log (1 / \varepsilon)\|a\|_{\infty}$.

Proof. We may clearly suppose $\|a\|_{\infty} \leq 1$. Define the BMOA function $A=a+i \mathscr{H}[a]$ and the outer function $\tau$

$$
\tau(z)=\exp \left\{\int_{\pi} \log \alpha(\theta) \frac{e^{i \theta}+z}{e^{i \theta}-z} m(d \theta)\right\} \quad \text { where } \alpha^{-1}=\max (1, \delta|A|) .
$$

Since $\tau=\alpha \exp i \mathscr{H}[\log \alpha]$ on $\pi$, it follows that for $|z|=1$

$$
|\tau A|=\alpha|A| \leq \delta^{-1}
$$

and hence $b=\operatorname{Re} \tau A$ satisfies $\|b\|_{\infty}+\|\mathscr{H}[b]\|_{\infty} \leq \sqrt{2} \delta^{-1}$. Also

$$
|a-b| \leq|1-\operatorname{Re} \tau||a|+|\operatorname{Im} \tau||A|
$$

implying

$$
\begin{aligned}
\|a-b\|_{2} & \leq\|1-\alpha \cos \mathscr{H}[\log \alpha]\|_{2}+\delta^{-1}\|\sin \mathscr{H}[\log \alpha]\|_{2} \\
& \leq\|1-\alpha\|_{2}+2 \delta^{-1}\|\mathscr{H}[\log \alpha]\|_{2} \\
& \leq m\left[|A|>\delta^{-1}\right]^{1 / 2}+2 \delta^{-1}\left\{\int_{\left[|A|>\delta^{-1}\right]}(\log \delta|A|)^{2}\right\}^{1 / 2}
\end{aligned}
$$

Since $m[|A|>\lambda] \leq c^{-1} e^{-c \lambda}$ for numerical $c>0$, the latter quantity is dominated by $c_{2} e^{-c / 3 \delta}$. Taking $\delta \sim(\log 1 / \varepsilon)^{-1}$, the lemma follows. 
The next fact is a consequence of the proof of Th. 5.1 in [2].

LEMMA 2. Assume $a$ in $L^{\infty}(\pi)$ taking values in $[-\pi, \pi[$ and $a=0$ outside some measurable set $U$ of $\pi$. Then, for given $\varepsilon>0$, there are Blaschke products $B_{1}, B_{2}$ fulfilling

$$
\left\|a-\operatorname{Arg} \frac{B_{1}}{B_{2}}\right\|_{\infty}<\varepsilon \text { and } \sum_{B_{1}(z) B_{2}(z)=0}(1-|z|)<\frac{c_{2}}{\varepsilon} m(U) .
$$

The method consists in covering $U$ by a countable family of disjoint intervals which union has approximately the same measure as $U$ and then starting consecutive approximations in $L^{1}$-norm using Lemma 5.5 of [2]. The construction yields moreover that the zeros of $B_{1}, B_{2}$ form an interpolating sequence, which will, however, not be used here.

Proof of the Proposition. We make an iteration construction which is again in the spirit of the proof of Theorem 5.1 of [2]. Starting from $a$ in $L^{\infty}(\pi)$ with values in $[-\pi, \pi$, a first application of Lemma 2 permits to replace $a$ by a function of small $L^{\infty}$-norm. We show now that if $a$ in $L_{R}^{\infty}(\pi)$ satisfies $\|a\|_{\infty}<\gamma(\gamma>0$ sufficiently small $)$, there is a decomposition

$$
a=b+\operatorname{Arg} \frac{B_{1}}{B_{2}}+a_{1} \quad\left(a_{1}, b \text { real }\right)
$$

where

(i) $\|b\|_{\infty}+\|\mathscr{H}[b]\|_{\infty} \leq c_{3}(\log 1 / \gamma) \gamma$

(ii) $\sum_{B_{1}(z) B_{2}(z)=0}(1-|z|) \leq c_{3} \gamma$

(iii) $\left\|a_{1}\right\|_{\infty} \leq 2 \gamma^{2}$.

Iteration provides then the required Blaschke products $B_{1}^{\prime}, B_{2}^{\prime}$ in the form $B_{j}^{\prime}=\Pi_{s} B_{j}^{(s)}(j=1,2)$, where (ii) bounds $\sum_{B_{j}^{\prime}(z)=0}(1-|z|)$ by the sum of an obviously converging series. The difference

$$
a-\operatorname{Arg} \frac{B_{1}^{\prime}}{B_{2}^{\prime}}=\sum_{s=0}^{\infty}\left(a_{s}-\operatorname{Arg} \frac{B_{1}^{(s)}}{B_{2}^{(s)}}-a_{s+1}\right)=\sum b_{s}
$$

then has a bounded Hilbert transform in view of (i).

To prove the decomposition stated above, apply first Lemma 1 with $\varepsilon=\gamma^{4}$ to obtain $b$ satisfying

$$
\|b\|_{\infty}+\|\mathscr{H}[b]\|_{\infty}<4 c_{1}\left(\log \frac{1}{\gamma}\right) \gamma \quad \text { and } \quad\|a-b\|_{1}<\gamma^{5} .
$$


For $\gamma$ small, the function $a-b$ is still $]-\pi, \pi$ [ valued. Moreover, the set $U=\left[|a-b|>\gamma^{2}\right]$ has measure less than $\gamma^{3}$. Application of Lemma 2 to the function $f=(a-b) \chi_{U}$ gives Blaschke products $B_{1}$ and $B_{2}$ so that

$$
\left\|f-\operatorname{Arg} \frac{B_{1}}{B_{2}}\right\|_{\infty}<\gamma^{2} \text { and } \sum_{B_{1}(z) B_{2}(z)=0}(1-|z|)<c_{2} \gamma \text {. }
$$

Put $a_{1}=a-b-\operatorname{Arg}\left(B_{1} / B_{2}\right)$, then $\left\|a_{1}\right\|_{\infty}<2 \gamma^{2}$, completing the proof.

\section{REFERENCES}

[1] R. G. Douglas and W. Rudin, Approximation by inner function, Pacific J. Math., 31, No. 2, (1969), 313-320.

[2] J. Garnett, Bounded Analytic Functions, Academic Press, 1967.

[3] P. Jones, Ratios of interpolating Blaschke products, Pacific J. Math., in press.

Received April 10, 1984 and in revised form September 12, 1984.

VRIJE UNIVERSITEIT BRUSSEL

PleinlaAn 2, 10F

1050 BRUSSELS, BeLgium 


\section{PACIFIC JOURNAL OF MATHEMATICS EDITORS}

V. S. VARADARAJAN (Managing Editor)
University of California
Los Angeles, CA 90024
HEBERT CLEMENS
University of Utah
Salt Lake City, UT 84112
CHARLES R. DEPRIMA
California Institute of Technology
Pasadena, CA 91125

\section{R. FINN}

Stanford University

Stanford, CA 94305

HeRmanN FlaschKa

University of Arizona

Tucson, AZ 85721

RAMESH A. GANGOLli

University of Washington

Seattle, WA 98195

ROBION KIRBY

University of California

Berkeley, CA 94720

\section{C. MoOre}

University of California

Berkeley, CA 94720

H. SAMELSON

Stanford University

Stanford, CA 94305

HAROLD STARK

University of California, San Diego

La Jolla, CA 92093

ASSOCIATE EDITORS
R. ARENS
E. F. BECKENBACH
B. H. NeUmanN
F. WOLF
K. Yoshida (1906-1982)

\section{SUPPORTING INSTITUTIONS}

UNIVERSITY OF ARIZONA

UNIVERSITY OF BRITISH COLUMBIA

CALIFORNIA INSTITUTE OF TECHNOLOGY

UNIVERSITY OF CALIFORNIA

MONTANA STATE UNIVERSITY

UNIVERSITY OF NEVADA, RENO

NEW MEXICO STATE UNIVERSITY

OREGON STATE UNIVERSITY
UNIVERSITY OF OREGON

UNIVERSITY OF SOUTHERN CALIFORNIA

STANFORD UNIVERSITY

UNIVERSITY OF HAWAII

UNIVERSITY OF TOKYO

UNIVERSITY OF UTAH

WASHINGTON STATE UNIVERSITY

UNIVERSITY OF WASHINGTON 


\section{Pacific Journal of Mathematics}

\section{Vol. 121, No. $1 \quad$ November, 1986}

Om P. Agrawal, Douglas Napier Clark and Ronald George Douglas,

Invariant subspaces in the polydisk $\ldots \ldots \ldots \ldots \ldots \ldots \ldots \ldots \ldots \ldots$

Christoph Bandt and Gebreselassie Baraki, Metrically invariant measures

on locally homogeneous spaces and hyperspaces $\ldots \ldots \ldots \ldots \ldots \ldots \ldots$

Marcy Mason Barge, Horseshoe maps and inverse limits ..............29

Russell Gene Bilyeu, Robert Richard Kallman and Paul Weldon Lewis,

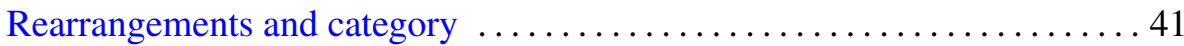

Jean Bourgain, A problem of Douglas and Rudin on factorization . . .......47

Hernan Cendra, A normal form and integration in finite terms for a class of

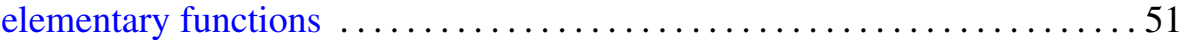

Ky Fan, The angular derivative of an operator-valued analytic function . . . . 67

Gerhard Gierz, On the Dunford-Pettis property of function modules of

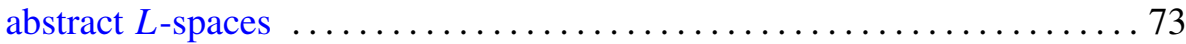

Gabriel Katz, On polynomial generators in the algebra of complex functions

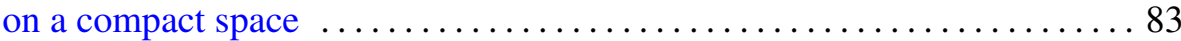

Ridgley Lange, Duality and asymptotic spectral decompositions $\ldots \ldots \ldots . .93$

Anthony To-Ming Lau and Peter F. Mah, Quasinormal structures for certain spaces of operators on a Hilbert space ................... 109

R. Daniel Mauldin, Correction: "The set of continuous nowhere differentiable functions"

Alan Harvey Mekler and Saharon Shelah, $\omega$-elongations and Crawley's problem

Alan Harvey Mekler and Saharon Shelah, The solution to Crawley's problem

Richard Rochberg, Deformation of uniform algebras on Riemann surfaces

Joseph Roitberg, On weak epimorphisms in homotopy theory

Jesús M. Ruiz, A remark on fields with the dense orbits property

Henry Wente, Counterexample to a conjecture of H. Hopf

David G. Wright, Rigid sets in $E^{n}$ 